\title{
SUMMING THROUGH TRIANGLE
}

\section{R. SIVARAMAN}

Associate Professor, Department of Mathematics, D. G. Vaishnav College, Chennai, India

ABSTRACT
Determination of sum of powers of natural numbers has been a quest for mathematicians from ancient times. The
formulas for sum of powers of natural numbers are widely used in deep research problems occurring in number theory
and combinatorics. In this paper, I discuss the construction of a triangle which provides the sums of powers of natural
numbers almost immediately.
KEYWORDS: Sum of Powers of Natural Numbers, Faulhaber Triangle, Bernoulli Numbers, Rightmost Diagonal
Entries, Harmonic Numbers

Received: Jun 09, 2020; Accepted: Jun 29, 2020; Published: Jun 30, 2020; Paper Id.: IJMPERDJUN2020291

\section{INTRODUCTION}

Ancient Greek mathematician Nicomachus has determined a nice formula for finding sum of squares of natural numbers. Several other Greek mathematicians have found ways to compute sum of powers of natural numbers up to third powers. In particular, Pythagoreans used these numbers to study broad class of numbers called Figurate numbers. It is quite amusing to note that the sums of powers of natural numbers are used even in the construction of magnificent structures like Pyramids and other great structures. During early part of seventeenth century, German mathematician Johann Faulhaber proposed a general technique to derive sum of powers of natural numbers. In this paper, we witness a novel method to determine sum of powers of natural numbers.

\section{Notations}

Let us denote sum of $k$ th powers of first $n$ natural numbers by

$$
S_{k}(n)=1^{k}+2^{k}+3^{k}+\cdots+n^{k}
$$

Our aim is to arrive a compact formula for determining the expression defined in (2.1). For doing this, we consider the construction of a triangle described below.

\section{Construction of Triangle}

We now construct a triangle of numbers whose entries are denoted by $T(p . q)$ where $q=0,1,2,3, \ldots, p$. Here $p$ denote the row beginning from 0 and $q$ denote the column beginning with 0 and ending with $p$ for given value of $p$. The entry of row 0 should be 1 . That is, $T(0,0)=1$. Assuming that row $p-1$ is known, the entries in the $p$ th row is given by the formula

$$
T(p, q)=T(p-1, q-1) \times \frac{p}{q+1}
$$


Equation (3.1) is used to compute $T(p, 1)$ up to $T(p, p)$.

The entries in the $p$ th row, first column are calculated in such a way that the row sum is always 1 . That is, we should have

$$
T(p, 0)=1-\sum_{q=1}^{p} T(p, q)
$$

Equations (3.1) and (3.2) are used to construct the following triangle up to first eleven rows.

\begin{tabular}{|c|c|c|c|c|c|c|c|c|c|c|c|}
\hline Row 0 & 1 & & & & & & & & & & \\
\hline \multirow{2}{*}{ Row 1} & 1 & 1 & & & & & & & & & \\
\hline & $\overline{2}$ & $\overline{2}$ & & & & & & & & & \\
\hline \multirow{2}{*}{ Row 2} & 1 & 1 & 1 & & & & & & & & \\
\hline & 6 & 2 & 3 & & & & & & & & \\
\hline \multirow{2}{*}{ Row 3} & & 1 & 1 & 1 & & & & & & & \\
\hline & 0 & $\overline{4}$ & $\overline{2}$ & $\overline{4}$ & & & & & & & \\
\hline \multirow{2}{*}{ Row 4} & -1 & & 1 & 1 & 1 & & & & & & \\
\hline & 30 & 0 & 3 & 2 & $\overline{5}$ & & & & & & \\
\hline \multirow{2}{*}{ Row 5} & & -1 & & 5 & 1 & 1 & & & & & \\
\hline & 0 & 12 & 0 & 12 & 2 & 6 & & & & & \\
\hline \multirow{2}{*}{ Row 6} & 1 & & -1 & & 1 & 1 & 1 & & & & \\
\hline & $\overline{42}$ & 0 & $\overline{6}$ & 0 & $\overline{2}$ & $\overline{2}$ & $\overline{7}$ & & & & \\
\hline \multirow[b]{2}{*}{ Row 7} & & 1 & & -7 & & 7 & 1 & 1 & & & \\
\hline & 0 & $\overline{12}$ & 0 & $\overline{24}$ & 0 & 12 & $\overline{2}$ & $\overline{8}$ & & & \\
\hline \multirow{2}{*}{ Row 8} & -1 & & $\underline{2}$ & & -7 & & $\frac{2}{2}$ & $\underline{1}$ & $\underline{1}$ & & \\
\hline & $\overline{30}$ & 0 & $\overline{9}$ & 0 & $\overline{15}$ & 0 & 3 & $\overline{2}$ & $\overline{9}$ & & \\
\hline \multirow{2}{*}{ Row 9} & & -3 & & 1 & & -7 & & 3 & 1 & 1 & \\
\hline & 0 & 20 & 0 & 2 & 0 & 10 & 0 & 4 & 2 & 10 & \\
\hline \multirow{2}{*}{ Row 10} & 5 & & -1 & & & & & & $\underline{5}$ & $\underline{1}$ & 1 \\
\hline & 66 & 0 & 2 & 0 & 1 & 0 & -1 & 0 & 6 & 2 & 11 \\
\hline
\end{tabular}

Col 0 Col 1 Col 2 Col 3 Col 4 Col 5 Col 6 Col 7 Col 8 Col 9 Col 10

Figure 1

The triangle in Figure 1 is called "Faulhaber Triangle" named in honour of the German mathematician Johann Faulhaber.

\section{Observations about Faulhaber's Triangle}

Assuming 1 as Row 0, Column 0 entry, and sum of enties in each row is always 1, we have constructed the Faulhaber triangle using the equations (3.1) and (3.2). With the above construction we notice the following properties:

The first column entries (except the second element 1/2) are the Bernoulli numbers. 
Theorem 1: The elements in row $p$, column $p$ is always $\frac{1}{p+1}$

Proof: To see this, from equation (3.1), we observe that

$$
\begin{aligned}
& T(p, p)=T(p-1, p-1) \times \frac{p}{p+1}=T(p-2, p-2) \times \frac{p-1}{p} \times \frac{p}{p+1}=\cdots \\
& =T(0,0) \times \frac{1}{2} \times \frac{2}{3} \times \cdots \times \frac{p-1}{p} \times \frac{p}{p+1}=1 \times \frac{1}{2} \times \frac{2}{3} \times \cdots \times \frac{p-1}{p} \times \frac{p}{p+1}=\frac{1}{p+1}
\end{aligned}
$$

The numbers in row $p$, column $p$ which we derived are called Harmonic numbers. These are the numbers $1, \frac{1}{2}, \frac{1}{3}, \frac{1}{4}, \frac{1}{5}, \cdots$ found in the rightmost diagonal of the triangle in Figure 1 .

Theorem 2: The elements in second rightmost diagonal (see Figure 1) is always $\frac{1}{2}$

Proof: This is because the elements in the second rightmost diagonal are located in row $p$, column $p-1$. Hence, from equation (3.1), we have

$$
T(p, p-1)=T(p-1, p-2) \times \frac{p}{p}=T(p-1, p-2)=T(p-1, p-2)=\cdots=T(1,0)=\frac{1}{2}
$$

Theorem 3: If there is an 0 entry in the triangle of Figure 1, then all the diagonal entries following it are also 0 . That is, if there is a 0 in row $p$, column $q$ then we should prove that the entry in row $(p+1)$ and column $(q+1)$ is also 0 .

Proof: To see this, we consider equation (3.1).to get

$$
T(p+1, q+1)=T(p, q) \times \frac{p+1}{q+2}=0 \times \frac{p+1}{q+2}=0
$$

Because of equation (3.1), if an entry in row $p$, column $q$ is positive then all subsequent entries following it in the rightmost diagonal are also positive. Similarly, if an entry is negative, then all subsequent entries following it in the rightmost diagonal are negative. The occurrence of both positive and negative terms in subsequent rows ensures the row sum to become 1 .

\section{Connection between Triangle and Summation}

In Figure 1 , we notice that there are $k+1$ entries in row $k$. If $c_{1}, c_{2}, \ldots, c_{k}, c_{k+1}$ are $k+1$ entries in row $k$ of Figure 1 in Faulhaber's Triangle, then the relation between Faulhaber Triangle and Summation is given by the following equation.

$$
S_{k}(n)=1^{k}+2^{k}+\cdots+n^{k}=c_{1} n+c_{2} n^{2}+\cdots+c_{k} n^{k}+c_{k+1} n^{k+1}
$$

where the coefficients $c_{1}, c_{2}, \ldots, c_{k}, c_{k+1}$ are $k$ th row entries of the Faulhaber's Triangle of Figure 1 read left to right. We notice from equations (4.1) and (4.2) that 


$$
c_{k+1}=T(k, k)=\frac{1}{k+1} \quad(5.2), \quad c_{k}=T(k, k-1)=\frac{1}{2}
$$

With the aid of equations (5.2) and (5.3), equation (5.1) becomes

$$
S_{k}(n)=1^{k}+2^{k}+\cdots+n^{k}=\frac{n^{k+1}}{k+1}+\frac{n^{k}}{2}+c_{k-1} n^{k-1}+c_{k-2} n^{k-2}+\cdots+c_{2} n^{2}+c_{1} n
$$

We now try to find other properties involved with the coefficients of equation (5.4).

Theorem 4: The coefficient $c_{k-1}$ is given by $c_{k-1}=\frac{k}{12}$

Proof: From Faulhaber's triangle in Figure 1, and equation (3.1), we see that

$$
\begin{aligned}
c_{k-1} & =T(k, k-2)=T(k-1, k-3) \times \frac{k}{k-1}=T(k-2, k-4) \times \frac{k-1}{k-2} \times \frac{k}{k-1}=T(k-2, k-4) \times \frac{k}{k-2} \\
& =T(k-3, k-5) \times \frac{k-2}{k-3} \times \frac{k}{k-2}=T(k-3, k-5) \times \frac{k}{k-3}=T(k-4, k-6) \times \frac{k}{k-4}=\cdots \\
& =T(2,0) \times \frac{k}{2}=\frac{1}{6} \times \frac{k}{2}=\frac{k}{12}
\end{aligned}
$$

Thus, we get equation (5.5) as required.

Theorem 5: The alternate coefficients $c_{k-2}, c_{k-4}, c_{k-6}, \ldots$ of equation (5.4) are zero.

Proof: First we observe that that these coefficients with respect to Figure 1 are given by

$$
c_{k-2}=T(k, k-3), c_{k-4}=T(k, k-5), c_{k-6}=T(k, k-7), \ldots
$$

Now, using equation (3.1), we get the following

$$
\begin{aligned}
c_{k-2} & =T(k, k-3)=T(k-1, k-4) \times \frac{k}{k-2}=T(k-2, k-5) \times \frac{k-1}{k-3} \times \frac{k}{k-2} \\
& =\cdots=T(3,0) \times \lambda_{1}=0 \times \lambda_{1}=0 \\
c_{k-4} & =T(k, k-5)=T(k-1, k-6) \times \frac{k}{k-4}=T(k-2, k-7) \times \frac{k-1}{k-5} \times \frac{k}{k-4} \\
& =\cdots=T(5,0) \times \lambda_{2}=0 \times \lambda_{2}=0
\end{aligned}
$$

Similarly, we get

$$
\begin{aligned}
& c_{k-6}=T(k, k-7)=T(7,0) \times \lambda_{3}=0 \times \lambda_{3}=0 \\
& c_{k-8}=T(k, k-9)=T(9,0) \times \lambda_{4}=0 \times \lambda_{4}=0
\end{aligned}
$$

Thus, we see that the alternate coefficients are all zero. 
We see that the alternating coefficients $c_{k-2 r}$ for $r=1,2,3,4, \ldots$ indeed are all zero because of the fact that they will reduce to the entry $\mathrm{T}(2 r+1,0)$ (see the diagonal leading to this entry in Figure 1), which is 0 in Figure 1 , for all natural numbers $r$. In fact, for more general equation, we have

$$
c_{k-2 r}=T(2 r+1,0) \times \lambda_{r}=0 \times \lambda_{r}=0
$$

This completes the proof.

\section{The Final Equations}

In view of Theorems 4 and 5, we can rewrite the formula for sum of powers of natural numbers in equation (5.4) to get the following equation

$$
S_{k}(n)=1^{k}+2^{k}+\cdots+n^{k}=\frac{n^{k+1}}{k+1}+\frac{n^{k}}{2}+\frac{k n^{k-1}}{12}+c_{k-3} n^{k-3}+c_{k-5} n^{k-5} \cdots+c_{2} n^{2}+c_{1} n
$$

If particular, if $k$ is odd (except $k=1$ ), then $k-2, k-4, k-6, \ldots$ are also odd, and so $c_{1}=0$. We thus see from equation (5.5) that all odd natural numbers $k$ (except $k=1$ ), $S_{k}(n)$ has $n^{2}$ as a factor and for all even natural numbers $k$, the sum of powers of natural numbers $S_{k}(n)$ has $n$ as a factor. With all these theorems and observations, we can now express the sum of powers of natural numbers for $k=0$ to 10 using Faulhaber Triangle in Figure 1 as follows:

$$
\begin{aligned}
& S_{0}(n)=1^{0}+2^{0}+\cdots+n^{0}=1 \times n=n \\
& S_{1}(n)=1^{1}+2^{1}+\cdots+n^{1}=\frac{1}{2} n^{2}+\frac{1}{2} n \\
& S_{2}(n)=1^{2}+2^{2}+\cdots+n^{2}=\frac{1}{3} n^{3}+\frac{1}{2} n^{2}+\frac{1}{6} n \\
& S_{3}(n)=1^{3}+2^{3}+\cdots+n^{3}=\frac{1}{4} n^{4}+\frac{1}{2} n^{3}+\frac{1}{4} n^{2} \\
& S_{4}(n)=1^{4}+2^{4}+\cdots+n^{4}=\frac{1}{5} n^{5}+\frac{1}{2} n^{4}+\frac{1}{3} n^{3}-\frac{1}{30} n \\
& S_{5}(n)=1^{5}+2^{5}+\cdots+n^{5}=\frac{1}{6} n^{6}+\frac{1}{2} n^{5}+\frac{5}{12} n^{4}-\frac{1}{12} n^{2} \\
& S_{6}(n)=1^{6}+2^{6}+\cdots+n^{6}=\frac{1}{7} n^{7}+\frac{1}{2} n^{6}+\frac{1}{2} n^{5}-\frac{1}{6} n^{3}+\frac{1}{42} n \\
& S_{7}(n)=1^{7}+2^{7}+\cdots+n^{7}=\frac{1}{8} n^{8}+\frac{1}{2} n^{7}+\frac{7}{12} n^{6}-\frac{7}{24} n^{4}+\frac{1}{12} n^{2} \\
& S_{8}(n)=1^{8}+2^{8}+\cdots+n^{8}=\frac{1}{9} n^{9}+\frac{1}{2} n^{8}+\frac{2}{3} n^{7}-\frac{7}{15} n^{5}+\frac{2}{9} n^{3}-\frac{1}{30} n \\
& S_{9}(n)=1^{9}+2^{9}+\cdots+n^{9}=\frac{1}{10} n^{10}+\frac{1}{2} n^{9}+\frac{3}{4} n^{8}-\frac{7}{10} n^{6}+\frac{1}{2} n^{4}-\frac{3}{20} n^{2} \\
& S_{10}(n)=1^{10}+2^{10}+\cdots+n^{10}=\frac{1}{11} n^{11}+\frac{1}{2} n^{10}+\frac{5}{6} n^{9}-n^{7}+n^{5}-\frac{1}{2} n^{3}+\frac{5}{66} n
\end{aligned}
$$

Note that these expressions confirm the facts and theorems that we had established in this paper. 


\section{CONCLUSIONS}

This paper provides a lucid way of summing powers of natural numbers to any desired number. For getting such formula, the entries of Figure 1, forms the coefficients and for given power, theorems 1 to 5 in sections 4 and 5 of this paper will immediately provide the idea to make up the equations quite easily. Thus using equation (3.1) and the theorems established in this paper, we can very easily determine sum of powers of natural numbers in quick time. Equation (5.5) provides the compact form of summing powrs of natural numbers. Deducing this formula is the main advantage of this paper. In doing so, as bonus we also encountered the most famous Bernoulli numbers as entries in column 0 of Figure 1. Though there are several other methods to arrive at the same equations described above, this is one of the best and most convenient method to find the sum of powers of natural numbers. It is also wonderful to see how simple relations such as in equation (3.1) can lead to so many wonderful observations and properties leading us to derive important equations. These equations have profound applications in higher branches of mathematics especially in Summability Theory and Analytic Number Theory.

\section{REFERENCES}

1. M. Bloom, An Old Algorithm for the sum of integer powers, Mathematics Magazine, Mathematical Association of America, Volume 66, (1993), pp. 304-305.

2. D. Knuth, Johann Faulhaber and sums of powers, Mathematics of Computation, Volume 61, (1993), pp. 277-294.

3. H. Sherwood, Sums of powers of integers and Bernoulli numbers, Mathematical Gazette, Volume 54, (1970), pp. $272-274$.

4. Mohammad Torabi-Dashti, Faulhaber's Triangle, The College Mathematics Journal, Volume 42, Issue 2, March 2011 , pp. 96-97.

5. R. Sivaraman, Summing Through Integrals, Science Technology and Development, Volume IX, Issue IV, April 2020, pp. 267272.

6. R. Sivaraman, Sum of powers of natural numbers, AUT AUT Research Journal, Volume XI, Issue IV, April 2020, pp. 353-359.

7. R. Sivaraman, Understanding Ramanujan Summation, International Journal of Advanced Science and Technology, Volume 29, No. 7, (2020), pp. 1472-485.

8. R. Sivaraman, Remembering Ramanujan, Advances in Mathematics: Scientific Journal, Volume 9, No.1, pp. 489-506.

9. G.F.C. de Bruyn \& J.M. de Villiers, Formulas for $1^{k}+2^{k}+3^{k}+\ldots+n^{k,}$ The Fibonacci Quarterly, Volume 32, Issue No. 3, (1994), pp. 271-276.

10. Joseph H. Silverman, A Friendly Introduction to Number Theory, Printice Hall Publications, Third Edition, 2006.

11. Rao, M. Ganeshwar. "ON (P, S)-Residue System Modulo N." International Journal of Mathematics and Computer Applications Research (IJMCAR) 4.2 (2014):69-74

12. Nanayakkara, Kadsa, and TSG Peiris. "Impact Of Mathematics In Level 1 on the Academic Performance of Engineering Students: A Case Study." International Journal of Applied Mathematics \& Statistical Sciences (IJAMSS) 5.4 (2016):1-8 
13. Asma, A. Faritha, And Ec Henry Amirtharaj. "Solution Of Single Objective Inventory Model Of Deteriorating Items With Fuzzy Cost Components As Triangular Fuzzy Numbers." International Journal Of Mathematics And Computer Applications Research (Ijmcar) 6.1 (2016):39-50

14. Pokharel, Jagat Krishna. "Effectiveness Of Teacher Training In Teaching Mathematics In Secondary Level Schools." International Journal Of Educational Science And Research (Ijesr) 8.4 (2018):9-14

15. Aboodh, K. S., et al. "Solving delay differential equations by Aboodh transformation method." International Journal of Applied Mathematics \& Statistical Sciences (IJAMSS) 7.2 (2018): 55-64. 
